\title{
Pseudo-spin Symmetry for Relativistic-Hyperbolic Problem and Tensor Potential
}

\author{
M. Eshghi * \\ Department of Physics, Central Tehran Branch, Islamic Azad University, Tehran, Iran
}

Received 23 July 2011, accepted in final revised form 15 August 2011

\begin{abstract}
We study the relativistic equation of spin-1/2 particles under the hyperbolic potential and a Coulomb-like tensor potential. By using the generalized parametric of the NikiforovUvarov method and the pseudo-spin symmetry, we obtain the energy eigenvalues equation and the corresponding unnormalized wave functions. Some numerical results are given, too.
\end{abstract}

Keywords: Dirac equation; Tensor potential; Pseudo-spin symmetry; Nikiforov-Uvarov.

(c) 2011 JSR Publications. ISSN: 2070-0237 (Print); 2070-0245 (Online). All rights reserved doi:10.3329/jsr.v3i3.8071 J. Sci. Res. 3 (3), $493-500$ (2011)

\section{Introduction}

The description of phenomena at high energies requires the investigation of relativistic wave equations. Dirac equation describes the dynamics of spin- $1 / 2$ particles $[1,2]$. The search for the exact solutions of the Dirac equation with various physical potentials plays an important role in nuclear physics and other related areas. Using the different method, some authors studied the bound solutions of the Dirac equation with these potentials [320]. The spin and pseudo-spin symmetry concepts introduced in nuclear theory [21, 22]. Spin symmetry occurs when scalar potential is nearly equal to the vector potential or equivalently $V_{v}(r) \cong V_{s}(r)$, in the nuclei while the pseudo-spin symmetry occurs when $V_{v}(r) \cong-V_{s}(r)$ [23]. The spin symmetry is relevant for mesons [24] and the pseudo-spin symmetry has been used to explain the features of deformed nuclei [25], superdeformation [26], and to establish an effective nuclear shell model scheme [21, 22]. Also, some researchers, various potentials such as the mie-type potential [27], Coulomb-like potential [28], Wood-Saxon potential [19], Eckart potential [29], etc., have been studied within the frame work of the spin and pseudo-spin symmetries.

On the other hand, tensor potentials were introduced into the Dirac equation with the substitution $\vec{p} \rightarrow \vec{p}-i m \omega \beta \cdot \hat{r} U(r)$. In this way, a spin-orbit coupling term is added to the Dirac Hamiltonian. In this regard, see [30-37].

The Hyperbolic potential model [38] and [39] is given by

*E-mail address: eshgi54@gmail.com 


$$
V(r)=D(1-\sigma \operatorname{coth} \alpha r)^{2}=\left(\frac{D_{1}+D_{2} e^{-2 \alpha r}}{1-e^{-2 \alpha r}}\right)^{2},
$$

where $D, \alpha$ and $\sigma$ are three positive parameters, and $D_{1}=\sqrt{D}(1-\sigma), D_{2}=\sqrt{D}(1+\sigma)$. The properties and applications of this potential are given in [38]. Recently, Jia et al. [40] have studied approximate analytical solutions of the Dirac equation with potential (1) under the conditions of spin and pseudo-spin symmetries. Ikhdair and Sever [41] have studied improved approximation to arbitrary l-state of the non-relativistic equation for this potential.

Our aim in this paper is to attempt to study the Dirac equation with hyperbolic potential (1) including a Coulomb-like tensor potential [30, 32]

$$
U(r)=-\frac{H}{r}, \quad H=\frac{Z_{a} Z_{b} e^{2}}{4 \pi \varepsilon_{0}}, \quad r \geq R_{c}
$$

(where $R_{c}=7.78 \mathrm{fm}$ is the Coulomb radius, $Z_{a}$ and $Z_{b}$ denote the charges of the projectile $a$ and the target nuclei $b$, respectively.) under the spin and pseudo-spin symmetries by using approximation scheme [44]. We obtain the energy eigenvalues equation and the corresponding spinor wave functions by using the parameteric generalized of the Nikiforov-Uvarov method $[32,45]$. This method has shown its power in calculating the exact energy levels for some solvable quantum systems.

\section{Dirac Equation with a Tensor Coupling}

According to the report given in the researcher [30-34], the Dirac equation with the scalar potential $V_{s}(r)$, vector potential $V_{v}(r)$ and a tensor potential $U(r)$ reads $(\hbar=c=1)$ :

$$
\left[\alpha \cdot P+\beta\left(M+V_{s}(r)\right)-i \beta \alpha \cdot \hat{r} U(r)\right] \psi_{n \kappa}(\vec{r})=\left[E-V_{v}(r)\right] \psi_{n \kappa}(\vec{r}),
$$

where $E$ is the relativistic energy of the system, $\vec{P}=-i \vec{\nabla}$ is the momentum operator, $\alpha$ and $\beta$ are the $4 \times 4$ matrices. In the relativistic quantum mechanics, $\left[\hat{H}, \hat{K}, \hat{J}^{2}, \hat{J}_{z}\right]$ can be taken as the complete set of the conservative quantities, the spin-orbit matrix operator $\hat{K}=-\beta(\hat{\sigma} . \hat{L}+1)$, where $L$ is orbital angular momentum of the spherical nucleons, commute with the Dirac Hamiltonian. Thus, the Dirac spinor can be written according to their angular momentum $j, \kappa$ and the radial quantum number $n$ and spin-orbit coupling quantum number $k$ as follows,

$$
\begin{aligned}
& \psi_{n \kappa}(\vec{r})=\frac{1}{r}\left[\begin{array}{l}
F_{n \kappa}(r) Y_{j m}^{l}(\theta, \varphi) \\
i G_{n \kappa}(r) Y_{j m}^{\tilde{l}}(\theta, \varphi)
\end{array}\right], \quad \kappa=\left(j+\frac{1}{2}\right) \\
& \psi_{n \kappa}(\vec{r})=\frac{1}{r}\left[\begin{array}{l}
F_{n \kappa}(r) Y_{j m}^{\tilde{I}}(\theta, \varphi) \\
i G_{n \kappa}(r) Y_{j m}^{\tilde{l}}(\theta, \varphi)
\end{array}\right], \quad \kappa=-\left(j+\frac{1}{2}\right)
\end{aligned}
$$


where $F_{n \kappa}(r)$ and $G_{n \kappa}(r)$ are the radial wave functions of the upper- and the lower-spinor components respectively, $Y_{j m}^{l}(\theta, \phi)$ and $Y_{j m}^{\tilde{I}}(\theta, \phi)$ are the spin and pseudo-spin spherical harmonics functions, respectively, the orbital and the pseudo-orbital angular momentum quantum numbers for spin symmetry $l$ and pseudo-spin symmetry $\tilde{l}$ refer to the upperand lower-component, respectively, $m$ is the projection of the total angular momentum on the $z$-axis.

Substituting (4) or (5) into (3), and using the following relations [2],

$$
\begin{aligned}
& (\vec{\sigma} \cdot \vec{A})(\vec{\sigma} \cdot \vec{B})=\vec{A} \cdot \vec{B}+i \vec{\sigma} \cdot(\vec{A} \times \vec{B}), \\
& (\vec{\sigma} \cdot \vec{P})=\vec{\sigma} \cdot \hat{r}\left(\hat{r} \cdot \vec{P}+i \frac{\vec{\sigma} \cdot \vec{L}}{r}\right),
\end{aligned}
$$

and properties

$$
\begin{aligned}
& (\vec{\sigma} . \vec{L}) Y_{m j}^{l}(\theta, \varphi)=(\kappa-\mathbb{D}) Y_{m j}^{\tilde{l}}(\theta, \varphi), \\
& (\vec{\sigma} \cdot \vec{L}) Y_{m j}^{\tilde{l}}(\theta, \varphi)=-(\kappa-\mathbb{D}) Y_{m j}^{l}(\theta, \varphi), \\
& (\vec{\sigma} . \hat{r}) Y_{m j}^{\tilde{l}}(\theta, \varphi)=-Y_{m j}^{l}(\theta, \varphi), \\
& (\vec{\sigma} . \hat{r}) Y_{m j}^{l}(\theta, \varphi)=-Y_{m j}^{\tilde{l}}(\theta, \varphi),
\end{aligned}
$$

yield two coupled differential equations as follows:

$$
\begin{aligned}
& \left(\frac{d}{d r}+\frac{\kappa}{r}-U(r)\right) F_{n \kappa}(r)=\left[E_{n \kappa}+M-\Delta(r)\right] G_{n \kappa}(r), \\
& \left(\frac{d}{d r}-\frac{\kappa}{r}+U(r)\right) G_{n \kappa}(r)=\left[M-E_{n \kappa}+\Sigma(r)\right] F_{n \kappa}(r),
\end{aligned}
$$

where $\Delta(r)=V_{v}(r)-V_{s}(r)$ and $\Sigma(r)=V_{v}(r)+V_{s}(r)$. By eliminating $G_{n \kappa}(r)$ in (12) and $F_{n \kappa}(r)$ in (13), we obtain two second-order differential equations for the upper and lower radial spinor components, respectively

$$
\begin{aligned}
& \left\{\frac{d^{2}}{d r^{2}}-\frac{\kappa(\kappa+1)}{r^{2}}+\frac{2 \kappa}{r} U(r)-\frac{d U(r)}{d r}-U^{2}(r)\right. \\
& \left.+\left(E_{n \kappa}+M-\Delta(r)\right)\left(E_{n \kappa}-M-\Sigma(r)\right)+\frac{\frac{d \Delta(r)}{d r}}{E_{n \kappa}+M-\Delta(r)}\left(\frac{d}{d r}+\frac{\kappa}{r}-U(r)\right)\right\} F_{n \kappa}(r)=0, \\
& \left\{\frac{d^{2}}{d r^{2}}-\frac{\kappa(\kappa-1)}{r^{2}}+\frac{2 \kappa}{r} U(r)+\frac{d U(r)}{d r}-U^{2}(r)\right.
\end{aligned}
$$




$$
\left.+\left(E_{n \kappa}+M-\Delta(r)\right)\left(E_{n \kappa}-M-\Sigma(r)\right)+\frac{\frac{d \Sigma(r)}{d r}}{M-E_{n \kappa}-\Sigma(r)}\left(\frac{d}{d r}-\frac{\kappa}{r}+U(r)\right)\right\} G_{n \kappa}(r)=0,
$$

where $\kappa(\kappa+1)=l(l+1)$ and $\kappa(\kappa-1)=\tilde{l}(\tilde{l}+1)$. The quantum number $\kappa$ is related to the quantum numbers for spin symmetry $l$ and pseudo-spin symmetry $\tilde{l}$ which is given as

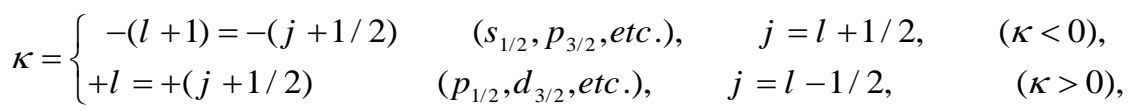

for $\kappa<0$ aligned spin and for $\kappa>0$ unaligned spin, and

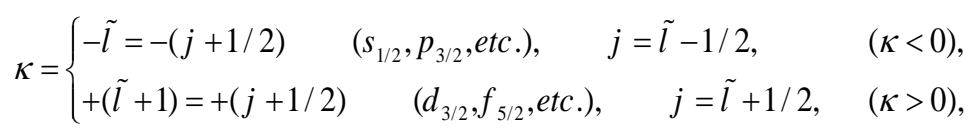

for $\kappa<0$ aligned pseudo-spin and for $\kappa>0$ unaligned pseudo-spin, and $\kappa= \pm 1, \pm 2, \ldots$.

\subsection{Pseudo-spin symmetry}

Substituting (1) and (2) into (15) and considering pseudo-spin symmetry (the condition of pseudo-spin symmetry $d \Sigma(r) / d r=0$ or $\Sigma(r)=$ const $\left.=C_{p s}\right)$ [42, 43], we obtain the second-order differential equation for the lower component of the Dirac spinor as follows,

$$
\left\{\frac{d^{2}}{d r^{2}}-\frac{(\kappa+H)(\kappa+H-1)}{r^{2}}+\left[E_{n \kappa}+M-D(1-\sigma \operatorname{coth} \alpha r)^{2}\right]\left(E_{n \kappa}-M-C_{p s}\right)\right\} G_{n \kappa}(r)=0 .
$$

Eq. (16) can not be solved analytically because of $(\kappa+H)(\kappa+H+1) / r^{2}$ term, therefore by using the approximation scheme suggested by Greene and Aldrich [44],

$$
\frac{1}{r^{2}} \approx \frac{\alpha^{2}}{\sinh ^{2} \alpha r}
$$

By using Eq. (17) and $s=e^{-2 \alpha r}, 0<s<1$, we have

$$
\left\{\frac{d^{2}}{d s^{2}}+\frac{1-s}{s(1-s)} \frac{d}{d s}+\frac{1}{s^{2}(1-s)^{2}}\left[-\xi_{1} s^{2}+\xi_{2} s-\xi_{3}\right]\right\} G_{n \kappa}(r)=0
$$

where

$$
\begin{array}{ll}
\xi_{1}=\frac{b_{2}}{4 \alpha^{2}}\left(D+D \sigma^{2}+2 D \sigma-b_{3}\right), & \xi_{2}=-b_{1}-\frac{b_{2}}{4 \alpha^{2}}\left(2 b_{3}+2 D+2 D \sigma^{2}\right), \\
\xi_{3}=\frac{b_{2}}{4 \alpha^{2}}\left(D+D \sigma^{2}+2 D \sigma-b_{3}\right) . &
\end{array}
$$


Following to the report given in the researcher [32, 45], we obtain the parameter set as,

$$
\begin{aligned}
& \alpha_{1}=1, \quad \alpha_{2}=1, \quad \alpha_{3}=1, \quad \alpha_{4}=0, \quad \alpha_{5}=-1 / 2, \\
& \alpha_{6}=\xi_{1},+1 / 4, \quad \alpha_{7}=-\xi_{2}, \quad \alpha_{8}=\xi_{3}, \quad \alpha_{9}=\xi_{1}-\xi_{2}+\xi_{3}+1 / 4 \text {, } \\
& \alpha_{10}=1+2 \sqrt{\xi_{3}}, \quad \alpha_{11}=2+2\left(\sqrt{\xi_{1}-\xi_{2}+\xi_{3}+1 / 4}+\sqrt{\xi_{3}}\right), \\
& \alpha_{12}=\sqrt{\xi_{3}}, \quad \alpha_{13}=-1 / 2-\left(\sqrt{\xi_{1}-\xi_{2}+\xi_{3}+1 / 4}+\sqrt{\xi_{3}}\right) \text {, }
\end{aligned}
$$

and we deduce the parameters required for the method [32, 45],

$$
\pi(s)=-\frac{1}{2} s \pm\left[\left(\xi_{1}+1 / 4-k\right) s^{2}+\left(-\xi_{2}+k\right) s+\xi_{3}\right]^{1 / 2},
$$

where

$$
k_{1,2}=-\left(-\xi_{2}+2 \xi_{3}\right) \pm 2 \sqrt{\xi_{3}\left(\xi_{1}-\xi_{2}+\xi_{3}+1 / 4\right)} .
$$

Different $k$ 's lead to the different $\pi$ 's. For

$$
k_{2}=-\left(-\xi_{2}+2 \xi_{3}\right)-2 \sqrt{\xi_{3}\left(\xi_{1}-\xi_{2}+\xi_{3}+1 / 4\right)},
$$

$\pi(s)$ becomes

$$
\pi(s)=-\frac{1}{2} s-\left[\left(\sqrt{\xi_{1}-\xi_{2}+\xi_{3}+1 / 4}+\sqrt{\xi_{3}}\right) s-\sqrt{\xi_{3}}\right] .
$$

We also have

$$
\tau(s)=1-2 s-2\left[\left(\sqrt{\xi_{1}-\xi_{2}+\xi_{3}+1 / 4}+\sqrt{\xi_{3}}\right) s-\sqrt{\xi_{3}}\right] .
$$

Therefore $\tau^{\prime}(s)<0$.

Following to the report given in the researcher [32, 45], we obtain the energy eigenvalues equation for the potential under the consideration

$$
n^{2}+n+\left(2 n+1+2 \sqrt{\xi_{3}}\right)\left(\sqrt{\xi_{1}-\xi_{2}+\xi_{3}+1 / 4}+\sqrt{\xi_{3}}\right)-\xi_{3}+1 / 2=0 .
$$

Some numerical results are given in Table 1, where we have used the parameters $C_{p s}=-1.05, M=7 \mathrm{fm}^{-1}, \alpha=0.32, D=0.88$ and $\sigma=0.0001$.

Table 1 . The bound state energy eigenvalues $E_{n k}$ in unit of $\mathrm{fm}^{-1}$ of the pseudo-spin symmetry hyperbolic potential for several values of $n$ and $k$.

\begin{tabular}{ccccccccc}
\hline$\tilde{l}$ & $n, \kappa<0$ & $l, j$ & $\begin{array}{c}E_{n, k<0} \\
(H=0)\end{array}$ & $\begin{array}{c}E_{n, k<0} \\
(H=5)\end{array}$ & $n-1, \kappa>0$ & $l+2, j+1$ & $\begin{array}{c}E_{n-1, k>0} \\
(H=0)\end{array}$ & $\begin{array}{c}E_{n-1, k>0} \\
(H=5)\end{array}$ \\
\hline 1 & $1,-1$ & $2 S_{1 / 2}$ & -5.90181 & -5.93506 & 0,2 & $4 d_{3 / 2}$ & -5.90181 & -6.02672 \\
2 & $1,-2$ & $3 p_{3 / 2}$ & -5.91524 & -5.91524 & 0,3 & $5 f_{5 / 2}$ & -5.91524 & -6.06382 \\
3 & $1,-3$ & $4 d_{5 / 2}$ & -5.93506 & -5.90181 & 0,4 & $6 g_{7 / 2}$ & -5.93506 & -6.09904 \\
4 & $1,-4$ & $5 f_{7 / 2}$ & -5.96079 & -5.89502 & 0,5 & $7 h_{9 / 2}$ & -5.96079 & -6.11982 \\
\hline
\end{tabular}


Now, let us give the corresponding upper Dirac spinor. We write the corresponding unnormalized eigenfunctions obtained in terms of the functions [32, 45],

$$
\rho(s)=s^{2 \sqrt{\xi_{3}}}(1-s)^{2 \sqrt{\xi_{1}-\xi_{2}+\xi_{3}+1 / 4}},
$$

and

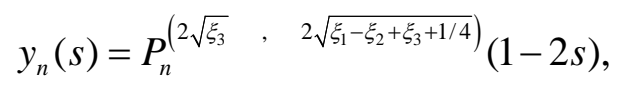

and

$$
\phi(s)=s^{\sqrt{\xi_{3}}}(1-s)^{1 / 2+\sqrt{\xi_{1}-\xi_{2}+\xi_{3}+1 / 4}}
$$

Now, let us give the corresponding lower Dirac spinor. By using [37], one obtains

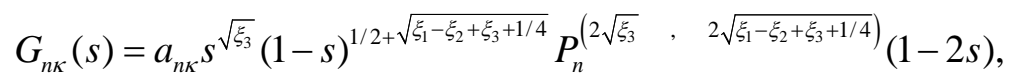

where $a_{n \kappa}$ is a normalization constant and it was determined by the condition

$$
\int_{-\infty}^{\infty} F_{n k}(s) d s=1 .
$$

where

$$
\xi_{1}-\xi_{2}+\xi_{3}=-b_{1}-\frac{b_{2} b_{3}}{\alpha_{2}}+\frac{D \sigma b_{2}}{\alpha_{2}}
$$

and

$$
b_{1}=(\kappa+H)(\kappa+H-1), \quad b_{2}=\left(E_{n \kappa}-M-C_{p s}\right), \quad b_{2}=E_{n \kappa}+M .
$$

\section{Discussion}

Choosing $\sigma=1$, Eq. (1) becomes

$$
V(r)=\frac{4 D e^{-4 \alpha r}}{\left(1-e^{-2 \alpha r}\right)^{2}} .
$$

Therefore, we can solve the Dirac equation by substituting $\sigma=1$ in Eqs. (16), (19), (31) for Eq. (33) under the pseudo-spin symmetry, also. With this form of the potential, we could obtain the energy eigenvalue equation and the corresponding eigenfunctions of the Dirac equation for the Manning-Rosen potential with appropriate placement [39, 41] in the equations. 


\section{Conclusion}

We have solved the Dirac equation with hyperbolic potential including a Coulomb-like tensor coupling in the case of spin and pseudo-spin symmetries. By using the approximation scheme and the parametric generalization of the Nikiforov-Uvarov method, we have obtained the energy eigenvalue equations and the corresponding unnormalized wave functions. Some numerical results were given for this potential.

\section{References}

1. W. Griner, Relativistic Quantum Mechanics-wave equation (Ed3), Springer-Verlag, Berlin, 2000).

2. J. D. Bjorken and S. D. Drell, Relativistic Quantum Mechanics (McGraw-Hill, NY, 1964).

3. C. S. Jia, T. Chen, L. G. Gui, Phys. Lett. A 373, 1621 (2009). http://dx.doi.org/10.1016/j.physleta.2009.03.006

4. Z. Fang, W. Yong, G. Jian-You, Commun. Theor. Phys. (Bijing, China), 52, 813 (2009). http://dx.doi.org/10.1088/0253-6102/52/5/09

5. C. L. Ho, Ann. Phys. 321, 2170 (2006). http://dx.doi.org/10.1016/j.aop.2005.12.005

6. H. Panahi and L. Jahangiry, Int. J. Theor. Phys. 48, 3234 (2009). http://dx.doi.org/10.1007/s10773-009-0126-7

7. A. D. Alhaidari, Phys. Lett. A 326, 58 (2004). http://dx.doi.org/10.1016/j.physleta.2004.04.031

8. M. R. Setare, S. Haidari, Int. J. Theor. Phys. 48, 3249 (2009). http://dx.doi.org/10.1007/s10773-009-0128-5

9. F. D. Adame and M. A. Gonzalez, Europhys. Lett. 13, 193 (1990). http://dx.doi.org/10.1209/0295-5075/13/3/001

10. A. S. de Castro and M. Hott, Phys. Lett. A 342, 53 (2005). http://dx.doi.org/10.1016/j.physleta.2005.05.039

11. S. Rukeng, Z. Yu, and H. Sizhu, Chin. Phys. Lett. 8, 114 (1991). http://dx.doi.org/10.1088/0256-307X/8/3/002

12. Y. Chargui, A. Trabelsi, and L. Chetouani, Phys. Lett. A 374, 531 (2010). http://dx.doi.org/10.1016/j.physleta.2009.11.028

13. A. S. de Castro, Ann. Phys. 311, 170 (2004). http://dx.doi.org/10.1016/j.aop.2003.12.007

14. V. M. Villalba, Il Nuovo Cimento 112B(1), 109 (1997).

15. C. Yan-Fu and D. Tong-Qing, Commun. Theor. Phys. 48, 431 (2007). http://dx.doi.org/10.1088/0253-6102/48/3/009

16. G. Jian-You, M. Jie, and X. Fu-Xin, Chin. Phys. Lett. 20, 602 (2003). http://dx.doi.org/10.1088/0256-307X/20/5/303

17. B. F. Samsonov and A. A. Pecheritsyn, Russ. Phys. J. 45, 13 (2002). http://dx.doi.org/10.1023/A:1016081217411

18. R. D. Benguria, H. Castillo, and M. Loewe, Revista Mexicana De Fisica 48 Supemento 3, 106 (2002).

19. J. Y. Guo and Z. Q. Sheng, Phys. Lett. A 338, 90 (2009). http://dx.doi.org/10.1016/j.physleta.2005.02.026

20. G. F. Wei and S. H. Dong, Phys. Lett. A 373, 2428 (2009). http://dx.doi.org/10.1016/j.physleta.2009.05.011

21. A. Arima, M. Harvey, and K. Shimizu, Phys. Lett. B 30, 517 (1969). http://dx.doi.org/10.1016/0370-2693(69)90443-2

22. K. T. Hecht and A. Adler, Nucl. Phys. A 137, 129 (1969). http://dx.doi.org/10.1016/0375-9474(69)90077-3

23. J. N. Ginocchio, Phys. Rev. Lett. 78, 436 (1997). http://dx.doi.org/10.1103/PhysRevLett.78.436

24. P. R. Page, T. Goldman, and J. N. Ginocchio, Phys. Lett. 86, 204 (2001). 
http://dx.doi.org/10.1103/PhysRevLett.86.204

25. A. Bohr, I. Hamamoto, and B. R. Mottelson, Phys. Scr. 26, 267 (1982).

http://dx.doi.org/10.1088/0031-8949/26/4/003

26. J. Dudek, W. Nazarewicz, Z. Szymanski, and G. A. Leander, Phys. Rev. Lett. 59, 1405 (1987). http://dx.doi.org/10.1103/PhysRevLett.59.1405

27. O. Aydogdu and R. Sever, Ann. Phys. 325, 373 (2010).

http://dx.doi.org/10.1016/j.aop.2009.10.009

28. S. M. Ikhdair and R. Sever, Appl. Math. Com. 46, 445 (2010).

29. L. H. Zhang, X. P. Li, and C. S. Jia, Phys. Lett. A 372, 2201 (2008). http://dx.doi.org/10.1016/j.physleta.2007.11.022

30. M. Eshghi and H. Mehraban, Few-Body Syst. (published online 11 June, 2011). doi:10.1007/s00601-011-0238-5

31. H. Akcay and C. Tezcan, Int. J. Mod. Phys. C 20, 931 (2009). http://dx.doi.org/10.1142/S0129183109014084

32. M. Hamzavi, H. Hassanabadi, and A. A. Rajabi, Int. J. Theor. Phys. (2010). http://dx.doi.org/10.1103/PhysRevA.14.2363

33. S. Zarrinkamar, A. A. Rajabi, and H. Hassanabadi, Ann. Phys. 325, 2522 (2010). http://dx.doi.org/10.1016/j.aop.2010.05.013

34. H. Akcay, Phys. Lett. A 373, 616 (2009). http://dx.doi.org/10.1016/j.physleta.2008.12.029

35. O. Aydogdu, and R. Sever, Few-Body Syst. 47, 193 (2010). http://dx.doi.org/10.1007/s00601-010-0085-9

36. M. Hamzavi, A. A. Rajabi, and H. Hassanabadi, Few-Bady Syst. 48, 171 (2010). http://dx.doi.org/10.1007/s00601-010-0095-7

37. S. M. Ikhdair and R. Sever, Appl. Math. Com. 216, 911 (2010). http://dx.doi.org/10.1016/j.amc.2010.01.104

38. D. Schioberg, Mol. Phys. 59 (5), 1123 (1986).

39. A. Arda, R. Sever, and C. Tezcan, Arxiv:0909.2086.

40. C. S. Jia, J. Y. Liu, P. Q. Wang, and X. Lin, Int. J. Theor. Phys. 48, 2633 (2009). http://dx.doi.org/10.1007/s10773-009-0051-9

41. S. M. Ikhdair and R. Sever, Arxiv:0809.2485

42. J. Meng, K. Sugawara-Tanaha, S. Yamaji, and A. Arima, Phys. Rev. C 59, 154 (1999). http://dx.doi.org/10.1103/PhysRevC.59.154

43. J. Meng, K. Sugawara-Tanaha, S. Yamaji, P. Ring, and A. Arima, Phys. Rev C 58, R628 (1998). http://dx.doi.org/10.1103/PhysRevC.58.R628

44. R. L. Greene and C. Aldrich, Phys. Rev. A 14, 2363 (1976). http://dx.doi.org/10.1103/PhysRevA.14.2363

45. C. Tezcan and R. Sever, Int. J. Theor. Phys. 48, 337 (2009). http://dx.doi.org/10.1007/s10773-008-9806-y 